\title{
Numerical Study of Buoyancy Convection of Air under Permanent Magnetic Field and Comparison with That under Gravity Field
}

\author{
Kewei Song, Wenkai Li, Yang Zhou, and Yuanru Lu \\ Department of Mechanical Engineering, Lanzhou Jiaotong University, Lanzhou, Gansu 730070, China \\ Correspondence should be addressed to Kewei Song; songkw@mail.lzjtu.cn
}

Received 9 May 2014; Revised 12 August 2014; Accepted 14 August 2014; Published 3 September 2014

Academic Editor: Jun Liu

Copyright (c) 2014 Kewei Song et al. This is an open access article distributed under the Creative Commons Attribution License, which permits unrestricted use, distribution, and reproduction in any medium, provided the original work is properly cited.

Magnetothermal free convection of air in a square enclosure under a nonuniform magnetic field provided by a permanent neodymium-iron-boron magnet is numerically studied. The natural convection under the gravity field alone is also studied for comparison. The physical fields of magnetizing force, velocity, and temperature as well as the local distribution characteristic of Nusselt number are all presented in this paper. The results show that the buoyancy convection of air in the square enclosure under magnetic field is quite different from that under the gravity field. The local value of Nusselt number under the magnetic field supplied by a permanent magnet with a residual magnetic flux density of about 4.5 Tesla can reach a high value of about three times larger than the maximum local value of Nusselt number under the gravity field. Relatively uniform distributions of temperature gradient and Nusselt number can be obtained along the cold wall of the enclosure under the magnetic field. A permanent magnet with high magnetic energy product with $B_{r}$ reaching to 3.5 Tesla can play a comparative role on the averaged Nusselt number compared with that under the gravity environment.

\section{Introduction}

The heat transfer and flow characteristics of natural convection in enclosures have attracted much research over the years due to their many practical engineering applications, such as in building insulation, growing crystals, solar energy collection, cooling of electrical industries, and flows in rooms due to thermal energy sources $[1,2]$. Natural convection flows in a vertical cavity with two vertical walls at different temperatures and with adiabatic horizontal surfaces are the most considered configuration in the studies of natural convection because of their relative simplicity and practical importance [3-5].

Fast development of superconducting materials at high temperature has enabled us to utilize commercial superconducting magnets that produce a magnetic flux density up to 10 Tesla or more. With the advent of superconducting magnets, magnetic convection can be studied on Earth, and various new findings have been reported in the last decades [6-10]. Wakayama [8-10] has been active in finding new and notable effects of a strong magnetic field in fluid convection. In the fields of fluid mechanics and heat transfer, the application of such high magnetic field on the control of natural convection has received considerable attention [11-21]. The heat transfer control of natural convection is based on the principle that the magnetizing force acts at a molecular level depending on the values of magnetic susceptibility of fluid. Therefore, the utilization of the magnetizing force is equivalent to the local gravity control within the region where the high gradient magnetic field exists. Braithwaite et al. [11] reported enhancement or cancellation of gravitational convection due to a magnetic field for a solution of gadolinium nitrate in a shadow layer heated from below and cooled from above. Tagawa et al. [12-20] carried out studies about the effects of magnetic fields on the natural convection. These studies have shown experimentally and numerically how to enhance or suppress heat transfer by the application of a magnetic field supplied by superconducting magnet.

Most of the previous studies about the magnetothermal natural convection in a vertical cavity focus on the effect of 


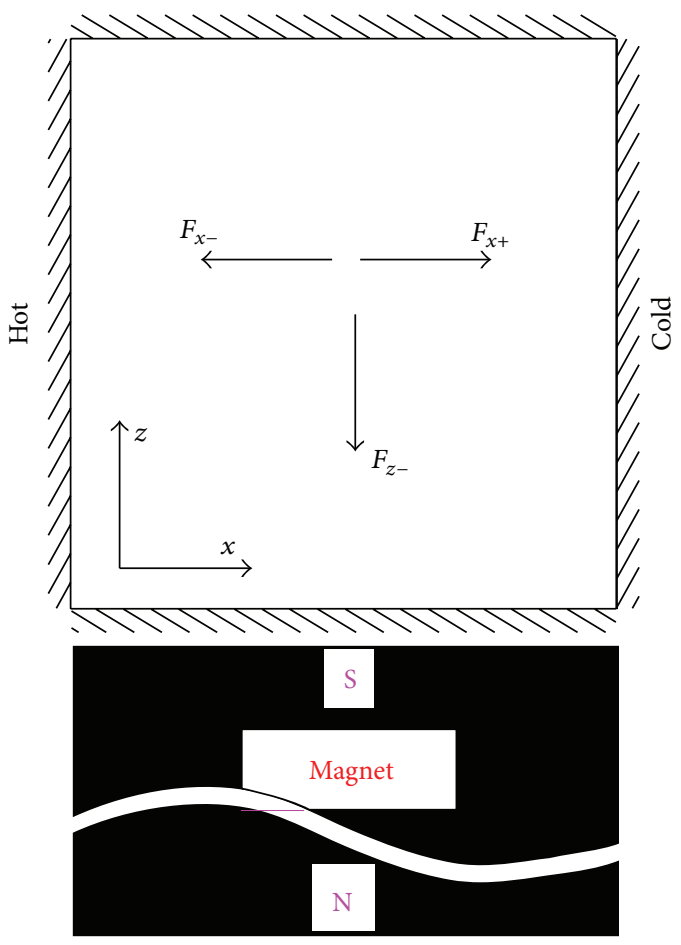

(a)

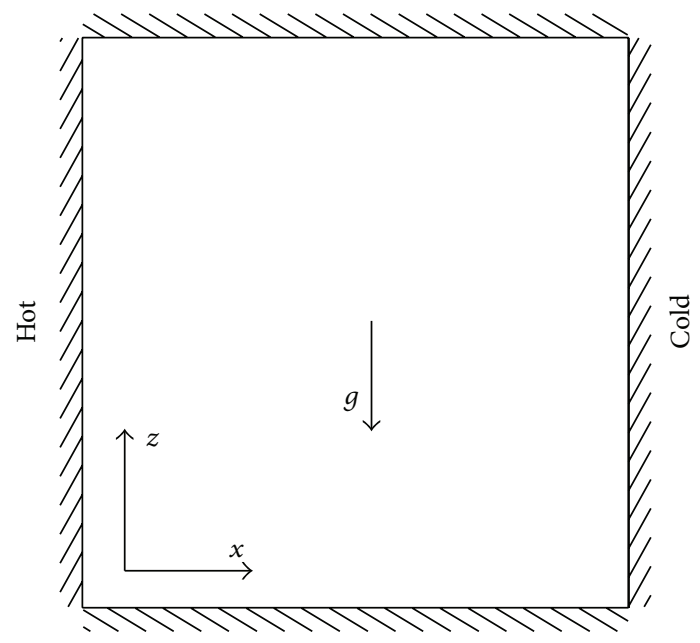

(b)

FIgURE 1: Configuration of simulation models. (a) Permanent magnetic field and (b) gravity field.

magnetic field supplied by superconducting magnet together with gravity field on natural convection. Bednarz et al. [21] studied the transient magnetic convection boundary layer in a microgravity environment. But the magnetic field supplied by the superconducting magnet is often assumed to be uniform; that is, the magnetic field gradient is constant. In this case, the problem under the uniform magnetic field is similar to that under gravity field. As the magnetic field supplied by permanent magnet is nonuniform and the gravity field is uniform, the natural convection characteristics under such two fields must be quite different. Recently, along with the development of the technology and improvement of manufacturing process, the residual magnetic flux density of a permanent magnet increases quickly. The maximum value of the residual magnetic flux density of a neodymium-ironboron magnet can reache to 1.5 Tesla or more. It is believed that the residual magnetic flux density of a permanent magnet will reach a larger value relying on the fast development of technology. The permanent magnet with large residual magnetic flux density can supply a nonuniform magnetic field and drive the gas free convection easily under a gradient temperature field. Thus, the permanent magnet which has high magnetic energy product can be widely applied in future for control of natural convection without any power supply from outside like a superconducting magnet.

The present study focuses on the heat transfer and flowing characteristic of the magnetothermal convection of air in a square enclosure driven by a permanent magnet field alone. Comparison is also carried out for the convection driven by magnetic field and gravity field, respectively.

\section{Physical Model}

The physical model, two-dimensional square enclosure, considered in this paper is shown in Figure 1. A cubic permanent magnet with the residual magnetic flux density in the $z$ direction is put on the bottom of the square enclosure. The length of both the magnet and square enclosure is $0.05 \mathrm{~m}$. There is a gap of $1 \mathrm{~mm}$ between the magnet and the square enclosure. Figures 1(a) and 1(b) show the physical model in which the left wall is heated and right wall is cooled isothermally, and the other two walls are thermally insulated. Figure 1(a) shows the physical model under gravity field; Figure 1(b) shows the model under magnetic field. The direction of magnetizing force and the gravity acceleration are also shown in Figure 1.

\section{Mathematical Equations}

In a single phase with isothermal state, both the magnetizing force and gravitational force are the conservative force and hence convection does not occur by itself no matter how strong it is. In order to arouse the convection, both the magnetic field gradient and the temperature gradient are necessary. In a normal expression, the magnetizing force is written by the equation as follows [22]:

$$
\mathbf{f}_{m}=\frac{\mu_{m}}{2} \chi \rho \nabla \mathbf{H}^{2} \cong \frac{1}{2 \mu_{m}} \rho \chi \nabla \mathbf{B}^{2} .
$$


The momentum equation including the magnetizing force alone is as follows:

$$
\rho\left[\frac{\partial \mathbf{u}}{\partial t}+(\mathbf{u} \cdot \nabla) \mathbf{u}\right]=-\nabla p+\mu \nabla^{2} \mathbf{u}+\frac{1}{2 \mu_{m}} \rho \chi \nabla \mathbf{B}^{2} .
$$

And the momentum equation including the gravity buoyancy force alone is

$$
\rho\left[\frac{\partial \mathbf{u}}{\partial t}+(\mathbf{u} \cdot \nabla) \mathbf{u}\right]=-\nabla p+\mu \nabla^{2} \mathbf{u}-\rho g .
$$

We assume that the air is an incompressible Newtonian fluid and the Boussinesq approximation is employed. When we consider the isothermal state, the fluid density $\rho$ and susceptibility $\chi$ would be constant, and convection does not arise in both the gravity and magnetic fields. Parameters under this state are $\rho_{0}, p_{0}$, and $\chi_{0}$ at reference temperature $T_{0}$. Hence we get

$$
\begin{gathered}
0=-\nabla p_{0}+\frac{1}{2 \mu_{m}} \rho_{0} \chi_{0} \nabla \mathbf{B}^{2} \\
0=-\nabla p_{0}-\rho_{0} g .
\end{gathered}
$$

When there is a temperature difference, the magnetic susceptibility and density also change with temperature. Subtracting (4) from (2) and (3) gives

$$
\begin{aligned}
& \begin{aligned}
\rho\left[\frac{\partial \mathbf{u}}{\partial t}\right. & +(\mathbf{u} \cdot \nabla) \mathbf{u}] \\
& =-\nabla\left(p-p_{0}\right)+\mu \nabla^{2} \mathbf{u}+\frac{\rho \chi-\rho_{0} \chi_{0}}{2 \mu_{m}} \nabla \mathbf{B}^{2} \\
\rho\left[\frac{\partial \mathbf{u}}{\partial t}\right. & +(\mathbf{u} \cdot \nabla) \mathbf{u}] \\
& =-\nabla\left(p-p_{0}\right)+\mu \nabla^{2} \mathbf{u}-\left(\rho-\rho_{0}\right) g .
\end{aligned}
\end{aligned}
$$

The density can be expressed by a Taylor expansion (keeping two terms only) around a reference state $T_{0}$ as follows:

$$
\rho=\rho_{0}+\left(\frac{\partial \rho}{\partial T}\right)_{0}\left(T-T_{0}\right)+\cdots .
$$

On the other hand, the thermal volume coefficient of expansion $\beta$ is defined for fluid as follows [23]:

$$
\beta=\frac{1}{V}\left(\frac{\partial V}{\partial T}\right)_{p}=\frac{1}{1 / \rho}\left(\frac{\partial 1 / \rho}{\partial T}\right)_{p}=-\frac{1}{\rho}\left(\frac{\partial \rho}{\partial T}\right)_{p} .
$$

Then, (6) can be written as follows:

$$
\rho-\rho_{0}=\left(\frac{\partial \rho}{\partial T}\right)_{0}\left(T-T_{0}\right)=-\rho_{0} \beta_{0}\left(T-T_{0}\right) .
$$

The difference of $\rho \chi$ can be written as

$$
\begin{aligned}
\rho \chi-(\rho \chi)_{0} & =\left(\frac{\partial \rho \chi}{\partial T}\right)_{0}\left(T-T_{0}\right) \\
& =\left(\chi \frac{\partial \rho}{\partial T}+\rho \frac{\partial \chi}{\partial T}\right)_{0}\left(T-T_{0}\right) .
\end{aligned}
$$

Magnetic susceptibility of air is a function of temperature; this follows the Curie law:

$$
\chi=\frac{C}{T} .
$$

Here, $C$ is a constant; then

$$
\frac{\partial \chi}{\partial T}=\frac{\partial}{\partial T}\left(\frac{C}{T}\right)=-\frac{\chi}{T} .
$$

The volumetric coefficient of expansion for an ideal gas can be given from the ideal gas law:

$$
p=\rho R_{g} T .
$$

Then, from (7)

$$
\begin{gathered}
\beta=-\frac{1}{\rho} \frac{\partial \rho}{\partial T}=-\frac{1}{\rho} \frac{\partial}{\partial T}\left(\frac{p}{R_{g} T}\right)=\frac{p}{\rho R_{g} T^{2}}=\frac{1}{T}, \\
\frac{\partial \rho}{\partial T}=-\rho \beta
\end{gathered}
$$

so that

$$
\begin{aligned}
\rho \chi-(\rho \chi)_{0} & =\left(-\chi \frac{\rho}{T}-\rho \frac{\chi}{T}\right)_{0}\left(T-T_{0}\right) \\
& =-2 \rho_{0} \beta_{0} \chi_{0}\left(T-T_{0}\right) .
\end{aligned}
$$

Given $p=p_{0}+p^{\prime}$, the momentum equation (5) can be written as follows:

$$
\begin{aligned}
{\left[\frac{\partial \mathbf{u}}{\partial t}\right.} & +(\mathbf{u} \cdot \nabla) \mathbf{u}] \\
& =-\frac{1}{\rho_{0}} \nabla p^{\prime}+\frac{\mu}{\rho_{0}} \nabla^{2} \mathbf{u}-\frac{\beta_{0} \chi_{0}\left(T-T_{0}\right)}{\mu_{m}} \nabla \mathbf{B}^{2} \\
{\left[\frac{\partial \mathbf{u}}{\partial t}\right.} & +(\mathbf{u} \cdot \nabla) \mathbf{u}] \\
& =-\frac{1}{\rho_{0}} \nabla p^{\prime}+\frac{\mu}{\rho_{0}} \nabla^{2} \mathbf{u}+g \beta_{0}\left(T-T_{0}\right) .
\end{aligned}
$$

Thus, the government equations for the natural convection caused by the magnetic buoyancy force or gravity buoyancy force are summarized as follows.

Continuity equation is

$$
\nabla \cdot \mathbf{u}=0 .
$$

Momentum equation, for magnetic field alone is

$$
\begin{aligned}
{\left[\frac{\partial \mathbf{u}}{\partial t}\right.} & +(\mathbf{u} \cdot \nabla) \mathbf{u}] \\
& =-\frac{1}{\rho_{0}} \nabla p^{\prime}+\frac{\mu}{\rho_{0}} \nabla^{2} \mathbf{u}-\frac{\beta_{0} \chi_{0}\left(T-T_{0}\right)}{\mu_{m}} \nabla \mathbf{B}^{2} .
\end{aligned}
$$

And for gravity field alone

$$
\begin{aligned}
{\left[\frac{\partial \mathbf{u}}{\partial t}\right.} & +(\mathbf{u} \cdot \nabla) \mathbf{u}] \\
& =-\frac{1}{\rho_{0}} \nabla p^{\prime}+\frac{\mu}{\rho_{0}} \nabla^{2} \mathbf{u}+g \beta_{0}\left(T-T_{0}\right) .
\end{aligned}
$$


Energy equation is

$$
\rho c_{p}\left[\frac{\partial T}{\partial t}+(\mathbf{u} \cdot \nabla) T\right]=\lambda \nabla^{2} T .
$$

In all the subsequent computation, the reference temperature $T_{0}$ is selected equal to the temperature of the cold wall $T_{c}$.

The initial condition $(t<0)$ is

$$
u=w=0, \quad T_{0}=T_{c} .
$$

Boundary conditions are

$$
\begin{gathered}
u=w=0, \quad \text { at } x=0, L, \quad \text { or } z=0, L \\
T=T_{h}, \quad \text { at } x=0 \\
T=T_{c}, \quad \text { at } x=L \\
\frac{\partial T}{\partial z}=0, \quad \text { at } z=0, L .
\end{gathered}
$$

The Rayleigh number is

$$
\mathrm{Ra}=\frac{\operatorname{Pr} \beta g\left(T_{h}-T_{c}\right) L^{3}}{v^{2}} .
$$

The local heat transfer coefficient $h_{\text {local }}$ is defined as

$$
h_{\text {local }}=\frac{\lambda}{T_{h}-T_{c}}\left|\frac{\partial T}{\partial x}\right| \text {. }
$$

The local Nusselt number $\mathrm{Nu}_{\text {local }}$ represents the ratio of heat transfer rate by convection to that by conduction in the fluid. $\mathrm{Nu}_{\text {local }}$ is given by

$$
\mathrm{Nu}_{\text {local }}=\frac{h_{\text {local }} L}{\lambda}
$$

The overall averaged Nusselt number is obtained by averaging $\mathrm{Nu}_{\text {local }}$ on the wall involved in heat transfer:

$$
\mathrm{Nu}_{m}=\frac{1}{L} \int_{L} \mathrm{Nu}_{\text {local }} d z
$$

In this study, the magnetic field is assumed to be provided by a cubic permanent neodymium-iron-boron (Nd-Fe-B) magnet which has a high magnetic energy product and could drive the gas free convection easily under a gradient temperature field. The magnetic field is solved using the vector potential method [24].

According to Maxwell's equations, the divergences of $\mathbf{B}$ is always zero:

$$
\nabla \cdot \mathbf{B}=0
$$

The fact that the divergence of $\mathbf{B}$ is zero means that there cannot exist sources of $\mathbf{B}$. Because the divergence of the curl of a vector is identically equal to zero. Thus the magnetic induction $\mathbf{B}$ can be expressed as the curl of a vector $\mathbf{A}$.

$$
\mathbf{B}=\nabla \times \mathbf{A} .
$$

A is the vector potential. The vector potential generated by the permanent magnet at the calculated point is as follows:

$$
\mathbf{A}=\frac{\mu_{0}}{4 \pi} \int_{v} \frac{\mathbf{M} \times \mathbf{r}_{1}}{r^{2}} d v
$$

Here, $v$ is the volume of the magnet, $r$ is the distance from the element volume $d v$ of the magnet to the point where the field is calculated, and $\mathbf{r}_{1}$ is the unit vector along $r$. When the calculated field point is fixed and the source point, that is the center of element volume $d v$, is moving, the gradient of $1 / r$ taken at the source point is

$$
\nabla\left(\frac{1}{r}\right)=\frac{\mathbf{r}_{1}}{r^{2}}
$$

Equation (28) can be rewritten as

$$
\mathbf{A}=\frac{\mu_{0}}{4 \pi} \int_{v} \mathbf{M} \times \nabla\left(\frac{1}{r}\right) d v .
$$

According to the curl calculus identities, the curl of the product of a scalar function $f$ and a vector function $\mathbf{C}$ is

$$
\begin{gathered}
\nabla \times(f \mathbf{C})=\nabla(f) \times \mathbf{C}+f(\nabla \times \mathbf{C}) \\
\nabla \times\left(\frac{\mathbf{M}}{r}\right)=\nabla\left(\frac{1}{r}\right) \times \mathbf{M}+\frac{1}{r}(\nabla \times \mathbf{M}) .
\end{gathered}
$$

And one of the properties of vector product is that the vector product is anticommutative:

$$
\nabla\left(\frac{1}{r}\right) \times \mathbf{M}=-\mathbf{M} \times \nabla\left(\frac{1}{r}\right) .
$$

Then,

$$
\mathbf{M} \times \nabla\left(\frac{1}{r}\right)=-\nabla \times\left(\frac{\mathbf{M}}{r}\right)+\frac{1}{r}(\nabla \times \mathbf{M}) .
$$

Equation (30) can be rewritten as

$$
\mathbf{A}=\frac{\mu_{0}}{4 \pi} \int_{v}\left(-\nabla \times\left(\frac{\mathbf{M}}{r}\right)+\frac{1}{r}(\nabla \times \mathbf{M})\right) d v .
$$

The curl calculus identity has

$$
\int_{v}\left(\nabla \times \frac{\mathbf{M}}{r}\right) d v=-\int_{S} \frac{\mathbf{M} \times \mathbf{n}}{r} d a,
$$

where $S$ is the surface bounding the volume $v$; $d a$ is element area of the surface $S, \mathbf{n}$ is the direction normal to the surface $S$. The magnetic vector potential can be rewritten as

$$
\mathbf{A}=\frac{\mu_{0}}{4 \pi} \int_{S} \frac{\mathbf{M} \times \mathbf{n}}{r} d a+\frac{\mu_{0}}{4 \pi} \int_{v} \frac{\nabla \times \mathbf{M}}{r} d v .
$$

$\mathbf{M} \times \mathbf{n}$ is the equivalent surface current density and $\nabla \times \mathbf{M}$ is the equivalent volume current density; the unit vector $\mathbf{r}_{1}$ points, from the source, which is the loop, to the point where the magnetic field is calculated. Here using the Biot-Savart law we can write down the value of $\mathbf{B}$ :

$$
\mathbf{B}=\frac{\mu_{0}}{4 \pi} \int_{S} \frac{\mathbf{M} \times \mathbf{n} \times \mathbf{r}_{1}}{r^{2}} d a+\frac{\mu_{0}}{4 \pi} \int_{v} \frac{\nabla \times \mathbf{M} \times \mathbf{r}_{1}}{r^{2}} d v .
$$




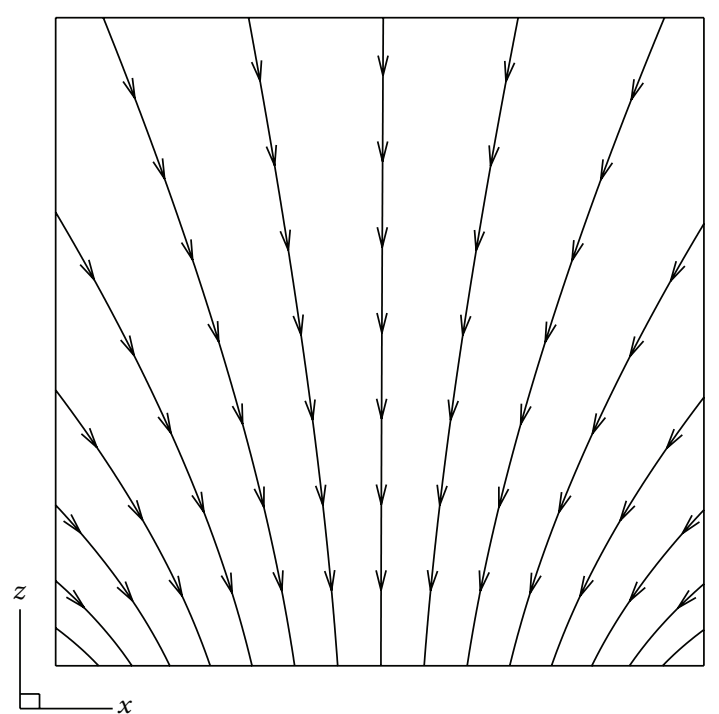

Figure 2: Magnetic field lines in the enclosure.

Thus, if we know the permanent magnet's magnetization $\mathbf{M}$, we can find the equivalent current densities. Then we can use the equivalent currents to calculate $\mathbf{B}$ by the upper equation.

In this study, the permanent magnet is applied near the bottom wall, and the poles of the magnet are lined parallel to the $Z$-direction. The square enclosure is placed on top of the cubic magnet in the middle of the $y$-side and parallel to the $X-Z$ plane. The computed magnetic field lines supplied by the cubic permanent magnet in the enclosure are shown in Figure 2.

The SIMPLE algorithm developed by Patankar [25] is used to solve the coupled heat transfer and fluid flow problem. The power-law scheme is used in the finite difference formulation of convection terms and a fine grid system is selected to raise the simulation precision. The grid-independence test for the solutions is carried out with three grid systems under the gravity field only. The averaged Nusselt numbers at different grid systems are presented in Table 1. Since the difference of Nusselt number between three grid systems is less than $0.5 \%$, the grid system $82 \times 82$ is selected for all computations. The physical properties of air and other constants used in this paper are summarized in Table 2.

\section{Results and Discussion}

All the computations are carried out from $\mathrm{Ra}=10^{4}$ to $\mathrm{Ra}=$ $10^{6}$, and the residual flux densities of permanent magnet changes from $0.5 \mathrm{~T}$ to $4.5 \mathrm{~T}$. The flow field, temperature field, and the distribution of local Nusselt number along the walls are obtained under the magnetic field with $B_{r}=4.5 \mathrm{~T}$; the difference of the effect of magnetic field and gravity field on natural convection is also studied.

4.1. Temperature Fields. Figure 3 shows the temperature fields of the studied physical models at $\mathrm{Ra}=10^{4}$ and $10^{5}$. For
TABLE 1: Grid independence tests $\left(\mathrm{Ra}=10^{5}, B_{r}=0\right)$.

\begin{tabular}{lc}
\hline Grid system & $\mathrm{Nu}$ \\
\hline $62 \times 62 \times 62$ & 4.540 \\
$82 \times 82 \times 82$ & 4.523 \\
$102 \times 102 \times 102$ & 4.520 \\
\hline
\end{tabular}

TABle 2: Parameters and constants ( $300 \mathrm{~K}, 1 \mathrm{~atm})$.

\begin{tabular}{lc}
\hline Acceleration coefficient of gravity $(g)$ & $9.8 \mathrm{~m} / \mathrm{s}^{2}$ \\
Prandtl number $(\operatorname{Pr})$ & 0.702 \\
Density of gas at $300 \mathrm{~K}(\rho)$ & $1.177 \mathrm{~kg} / \mathrm{m}^{3}$ \\
Kinematic viscosity of gas $(\nu)$ & $15.718 \times 10^{-6} \mathrm{~m}^{2} / \mathrm{s}$ \\
Viscosity of gas $(\mu)$ & $18.5 \times 10^{-6} \mathrm{~Pa} \cdot \mathrm{s}$ \\
Conduction coefficient $(\lambda)$ & $2.646 \times 10^{-2} \mathrm{~W} /(\mathrm{m} \cdot \mathrm{K})$ \\
Thermal diffusivity of gas $(\alpha)$ & $22.45 \times 10^{-6} \mathrm{~m}^{2} / \mathrm{s}$ \\
Mass magnetic susceptibility of & $1.738 \times 10^{-6} \mathrm{~m}^{3} / \mathrm{kg}$ \\
oxygen $\left(\chi_{\mathrm{O}_{2}}\right)$ & $300.0 \mathrm{~K}$ \\
Cold wall temperature $\left(T_{c}\right)$ & $1 / 300.0(1 / \mathrm{K})$ \\
$\begin{array}{l}\text { Volumetric coefficient of expansion } \\
\text { of gas }(\beta)\end{array}$ & $4 \pi \times 10^{-7} \mathrm{H} / \mathrm{m}$ \\
$\begin{array}{l}\text { Magnetic permeability of free space } \\
\left(\mu_{0}\right)\end{array}$ & \\
\hline
\end{tabular}

the physical model under the gravity field, as shown in Figure 3(a), the temperature field looks rotationally symmetric about the center of the enclosure. The temperature gradient normal to the hot wall decreases and the temperature gradient near the cold wall increases gently from the bottom wall to the top wall. The largest temperature gradient near the hot wall locates at the position close to the bottom wall, but near the cold wall it is close to the top wall. The temperature field structure under the magnetic field, as shown in Figure 3(b), is quite different from the temperature field under the gravity field. The temperature gradient near the hot and cold walls decreases along $z$-direction. The maximum gradient of the temperature near the hot wall locates at the bottom of the hot wall, and the maximum temperature gradient on the cold wall locates at the position close to the bottom wall. The temperature gradient normal to the cold wall changes a little from top wall to the position close to the bottom wall, and then it decreases quickly till to the bottom wall in a small range.

4.2. Magnetizing Force Fields. Figure 4 shows the gravity buoyancy force and magnetizing buoyancy force fields in the enclosure. From these figures we can find that the magnetizing buoyancy force is different from the gravity buoyancy force. The gravity buoyancy force parallels along $z$-direction, but the magnetizing buoyancy force's direction changes in the enclosure. The magnetizing buoyancy force increased with increase of magnet strength. There is large magnetizing force near the bottom wall of the enclosure and the force near the hot wall is larger than that near the cold wall. The magnetizing force decreases quickly along $z$-direction. But for the gravity field, the distribution of 


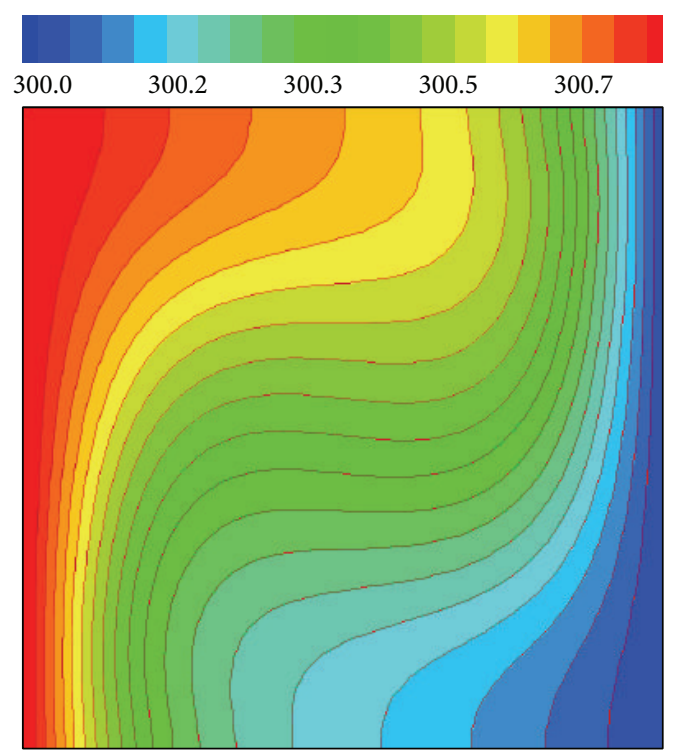

$\mathrm{Ra}=10^{4}$
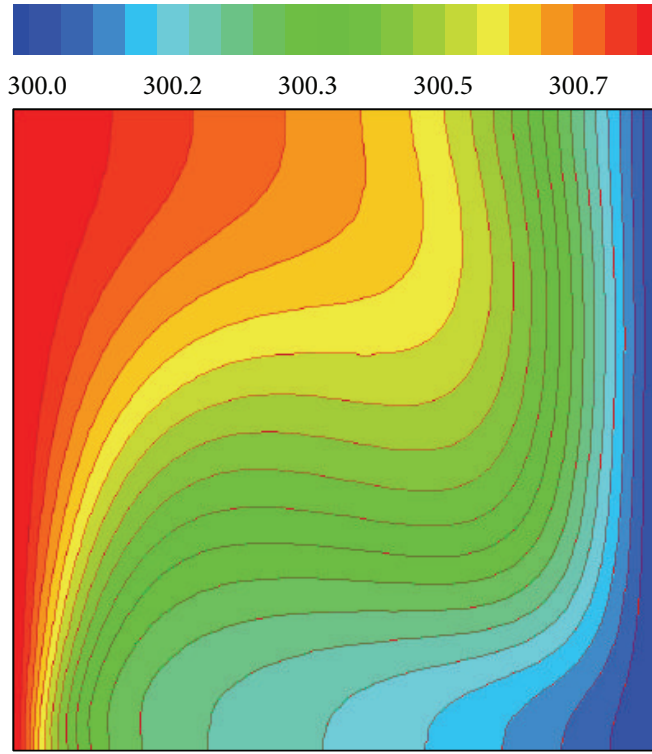

$\mathrm{Ra}=10^{4}$

(a)

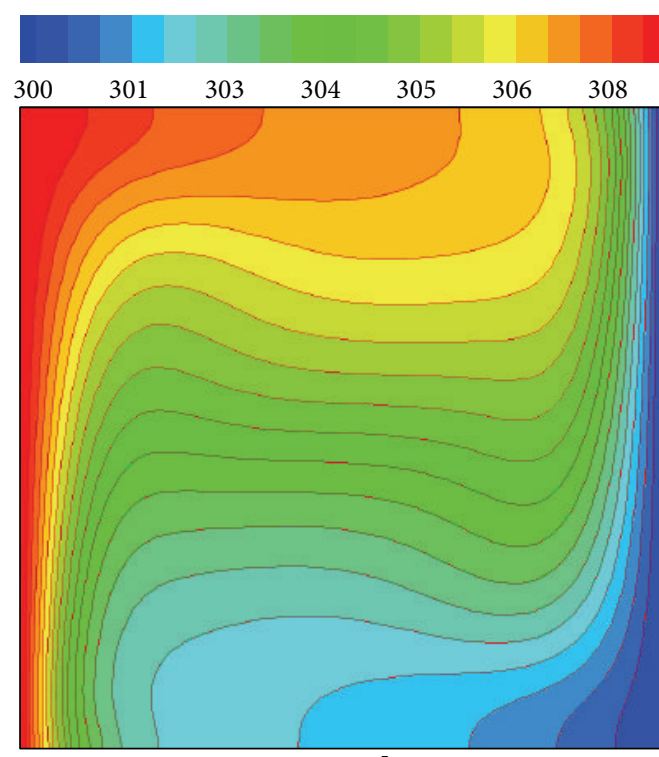

$\mathrm{Ra}=10^{5}$
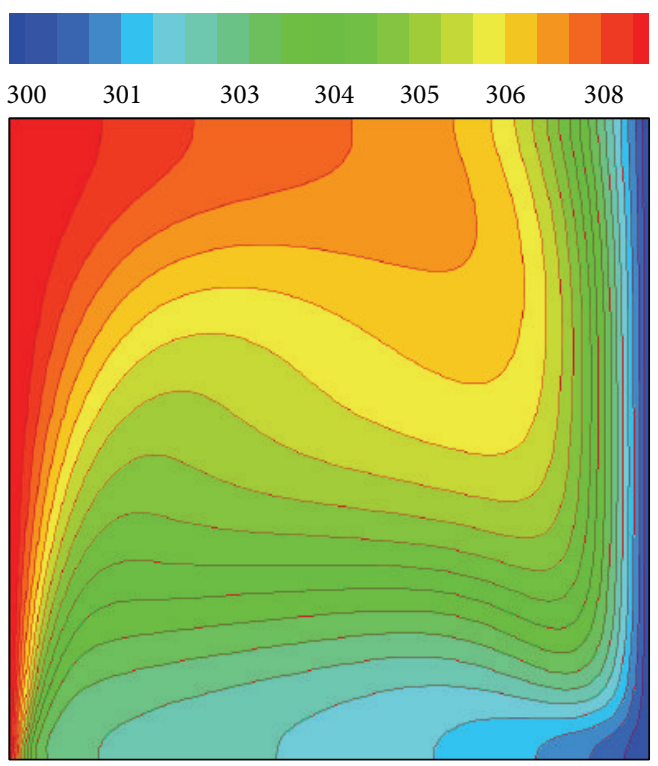

$\mathrm{Ra}=10^{5}$

(b)

Figure 3: Temperature fields at $\mathrm{Ra}=10^{4}$ and $10^{5}$. (a) Under gravity field and (b) under magnetic field, $B_{r}=4.5 \mathrm{~T}$.

gravity buoyancy force is quite different. The larger gravity buoyancy force locates in the region near the top and hot wall, and the gravity buoyancy force decreases from hot wall to cold wall and increases from bottom wall to top wall. The maximum value of magnetizing buoyancy force is larger than the maximum gravity buoyancy force.

4.3. Velocity Fields. Figure 5 shows the velocity fields under gravity field and magnetic field. When there is gravity field only, as shown in Figure 5(a), the large value of velocity locates near the walls and the value of velocity decreases from the walls to the center of the enclosure. There is one vortex in the center of the enclosure at $\mathrm{Ra}=10^{4}$. When $\mathrm{Ra}$ increases to $10^{5}$, the core of the vortex is divided into two cores, one is located near the top and hot walls, and the other one locates close to the cold and bottom walls. When there is magnetic field only, the velocity field is different from that under the gravity field. A vortex is also formed in the enclosure, but the core of the vortex locates close to the bottom wall at $\mathrm{Ra}=10^{4}$. When $\mathrm{Ra}$ increases to $10^{5}$, there is still only one core of the vortex and the core moves much closer to the hot wall. Figures 6(a) and 6(b) show the streamlines in the enclosure under gravity field and magnetic field, respectively. The streamlines related to gravity field look 


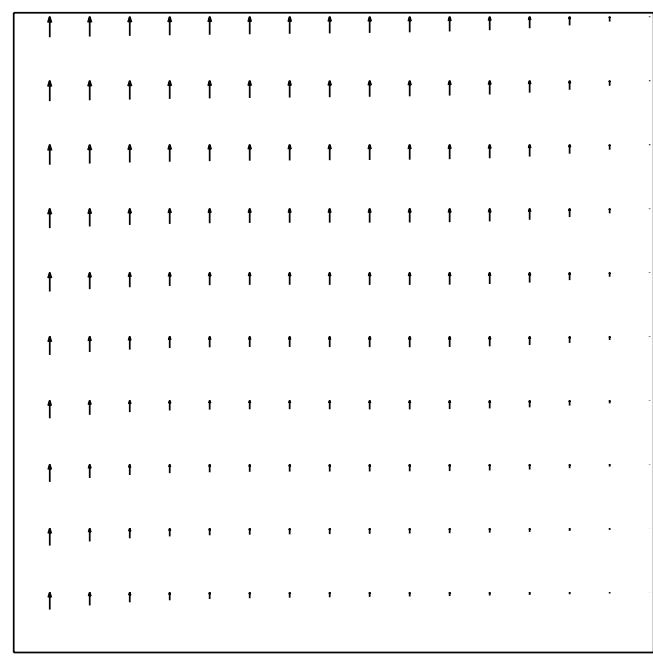

$\mathrm{Ra}=10^{4}$ $0.1 \mathrm{~N}$

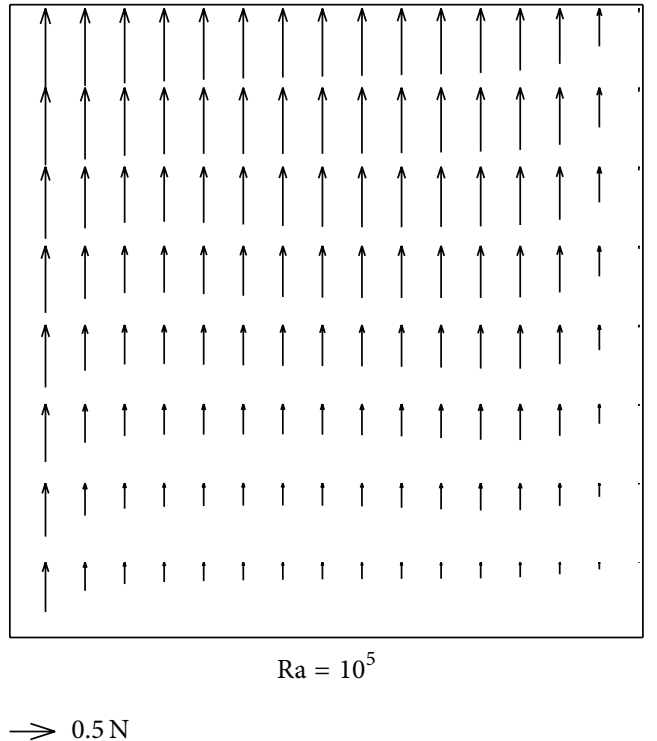

(a)
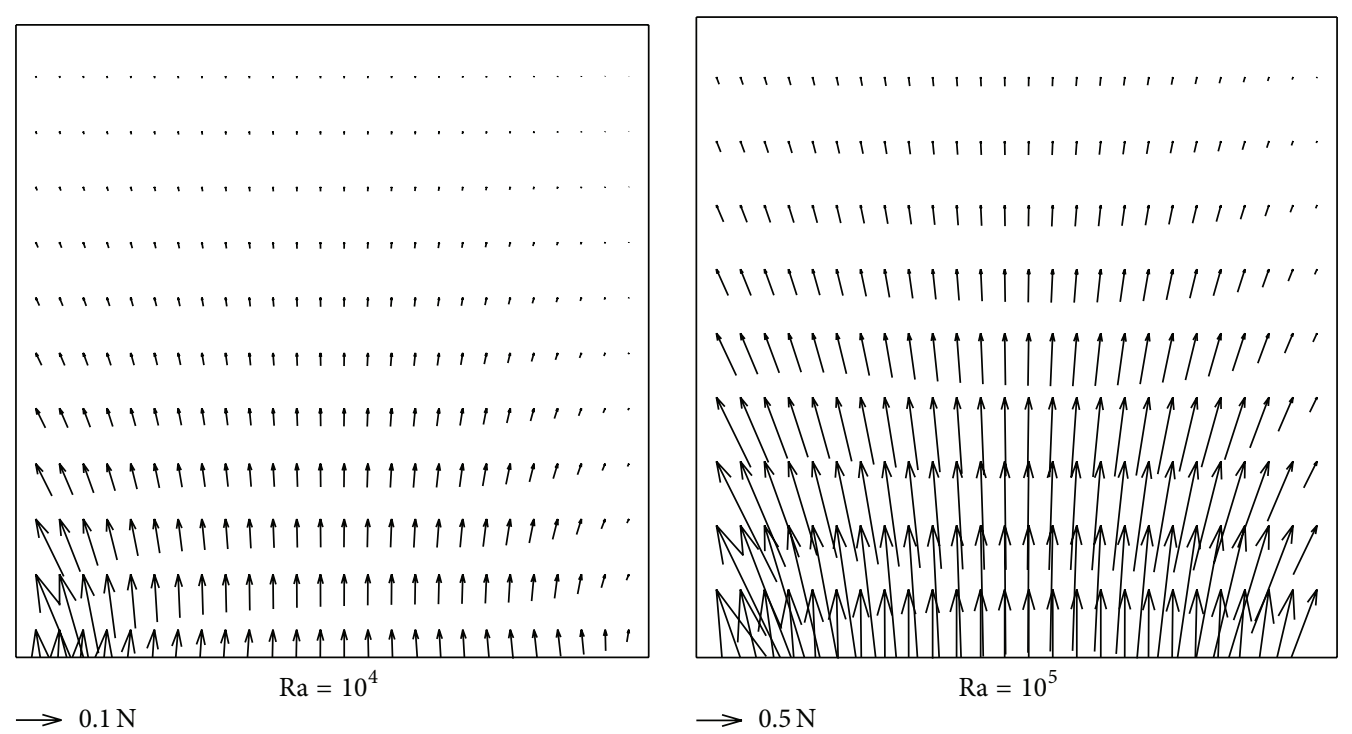

(b)

FIGURE 4: Gravity and magnetizing buoyancy force fields. (a) $g$ and (b) $B_{r}=4.5 \mathrm{~T}$.

rotationally symmetric about the center of the enclosure; large velocity gradient normal to the wall locates near the bottom segment of the hot wall and the top segment of the cold wall. But under the magnetic field, the streamlines are not symmetric and there are dense streamlines near the corner of the hot and bottom walls where there is large value of magnetizing force as shown in Figure 4(b). In the region near the top wall, the velocity gradient normal to the wall is significantly less than that in other regions.

4.4. Distribution of Temperature along Lines. The distribution curves of temperature at $\mathrm{Ra}=10^{5}$ along the lines parallel to $x$-axis are plotted in Figure 7; these three lines $z 1, z 2$, and $z 3$ are at the positions $z / L=0.1,0.5$, and 0.8 , respectively. In the region near the hot wall, the temperature under the magnetic field along the line $z 1$ is lower than that under the gravity field, but along the lines $z 2$ and $z 3$, the temperature under the magnetic field is higher than the temperature under the gravity field. In the region near the cold wall, the temperature related to magnetic field is higher than the temperature related to gravity field along the lines $z 1$ and $z 2$, and the temperature related to magnetic field is lower than the temperature related to the gravity field along line $z 3$. The high temperature near the cold wall means a high temperature gradient and high temperature near the hot wall means a low temperature gradient. For the magnetic field, the largest temperature gradient near the cold wall is along the line $z 2$ 


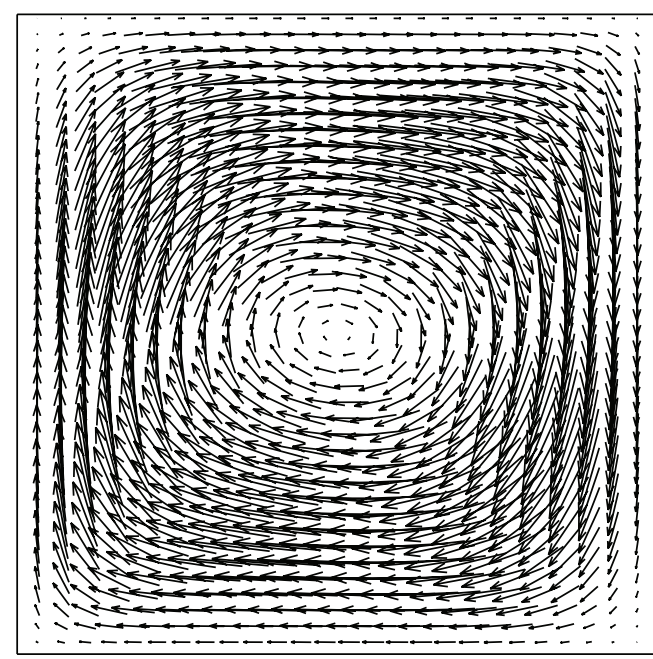

$\mathrm{Ra}=10^{4}$

$\longrightarrow 0.005 \mathrm{~m} / \mathrm{s}$

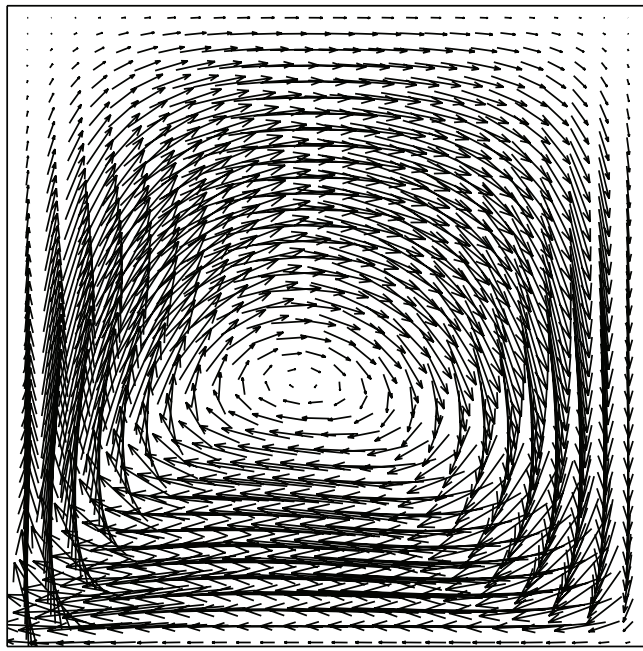

$\mathrm{Ra}=10^{4}$

$\longrightarrow 0.005 \mathrm{~m} / \mathrm{s}$

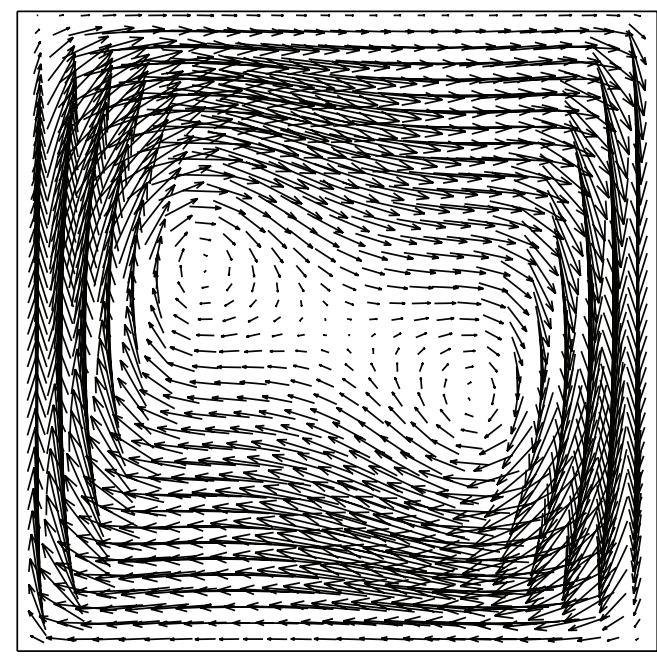

$\mathrm{Ra}=10^{5}$

(a)

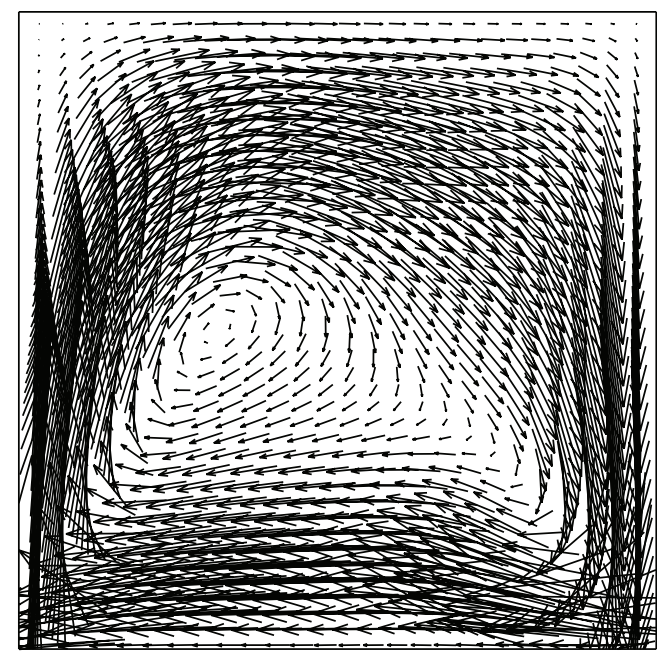

$\mathrm{Ra}=10^{5}$

$\longrightarrow 0.01 \mathrm{~m} / \mathrm{s}$

(b)

FIGURE 5: Comparison of velocity vectors of physical models. (a) Under gravity field and (b) under magnetic field.

and the largest temperature gradient near the hot wall is along the line $z 1$. We can obtain the same conclusion as in Figure 7 that the distribution of the temperature under the magnetic field is quite different from that under the gravity field.

4.5. Heat Transfer on the Walls. The distribution curves of the local Nusselt number along the hot and cold walls are plotted in Figure 8. The distribution of the local Nusselt number under gravity field is shown in Figure 8(a). The peak value along the hot wall locates near the bottom wall and decreases nearly linearly to the top wall. The distribution of local Nusselt number along the cold wall is symmetric about the central line of the enclosure with the distribution of local
Nusselt number along the hot wall. The value of the Nusselt number increases nearly linearly from bottom wall and gets the peak value near the top wall; then it decreases again till to the top wall. Figure 8(b) shows the distribution curves of local Nusselt number under the magnetic field with $B_{r}=$ 4.5 T. We can see that the distribution of the local Nusselt number for the model under magnetic field is different from that shown in Figure 8(a). The local Nusselt number gets a high peak value at the bottom of the hot wall and it decreases quickly for a short distance and then continues decreasing at a relatively slow rate till it reaches the top wall. The change of the local Nusselt number along the cold wall is much gentler compared with that along the hot wall. The local Nusselt 


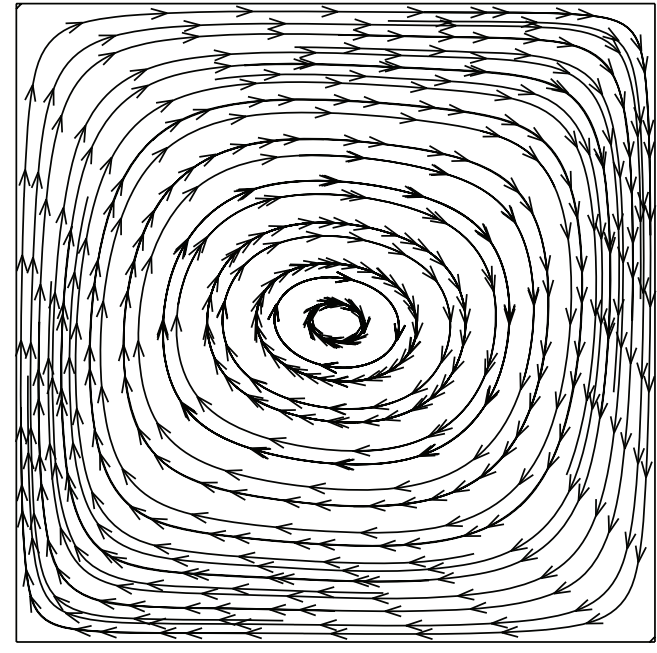

$\mathrm{Ra}=10^{4}$

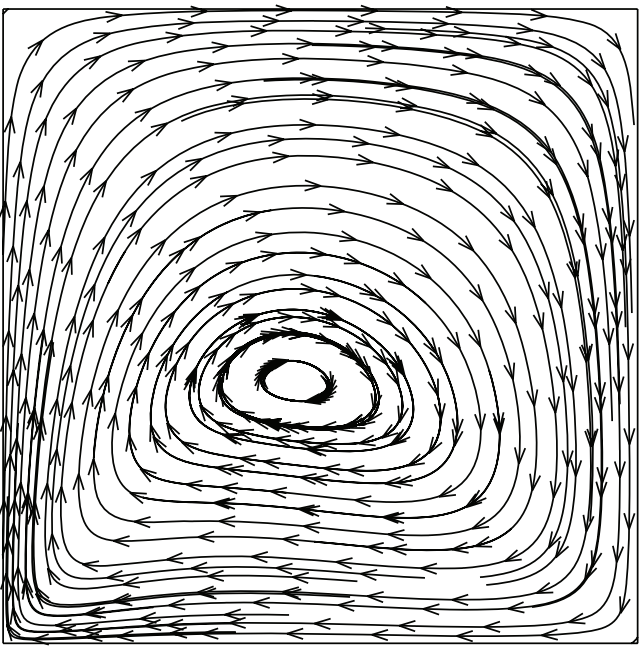

$\mathrm{Ra}=10^{4}$

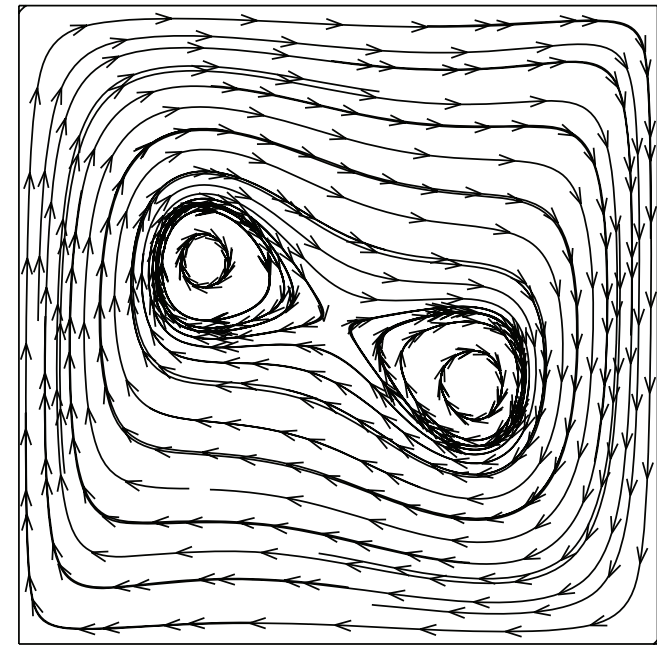

$\mathrm{Ra}=10^{5}$

(a)

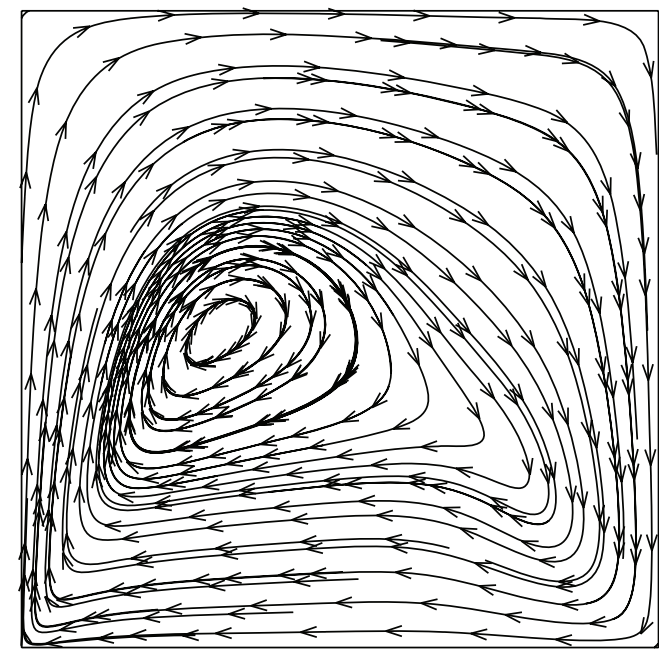

$\mathrm{Ra}=10^{5}$

(b)

FIGURE 6: Comparison of streamlines. (a) Under gravity field and (b) under magnetic field.

number increases slowly from the top wall to the position near the bottom wall and then it decreases quickly till it reaches the bottom wall.

The comparison of the distribution curves of the local Nusselt number under gravity field and magnetic field are presented in Figure 9. The value of local Nusselt number along the hot wall is larger than the value under the gravity filed in a short line segment from the bottom wall; the peak value of local Nusselt number related to magnetic field is more than three times larger than the peak value related to the gravity field. On the cold wall, the local Nusselt number related to magnetic field changes much more gently and has larger value on the lower part of the wall compared with that related to gravity field.

Figure 10 shows the distribution of the average Nusselt number with Ra ranging from $10^{4}$ to $10^{6}$ and the residual magnetic flux density of a permanent magnet ranging from $1.5 \mathrm{~T}$ to $4.5 \mathrm{~T}$. The average Nu under the magnetic field has a similar distribution curve compared with that under the gravity field. The averaged $\mathrm{Nu}$ increases with increase of $B_{r}$. The averaged Nusselt numbers on the hot wall with $\mathrm{Ra}=$ $10^{5}$ are summarized in Table 3. When $B_{r}$ is about 3.5T, the averaged $\mathrm{Nu}$ is nearly the same as that under gravity field; this means that the magnetizing buoyancy force has a comparative effect on $\mathrm{Nu}$ in the enclosure compared with the gravity buoyancy force.

\section{Conclusion}

Natural convection of air in a two-dimensional square enclosure under a nonuniform magnetic field provided by a permanent magnet is carried out. The results are compared with 


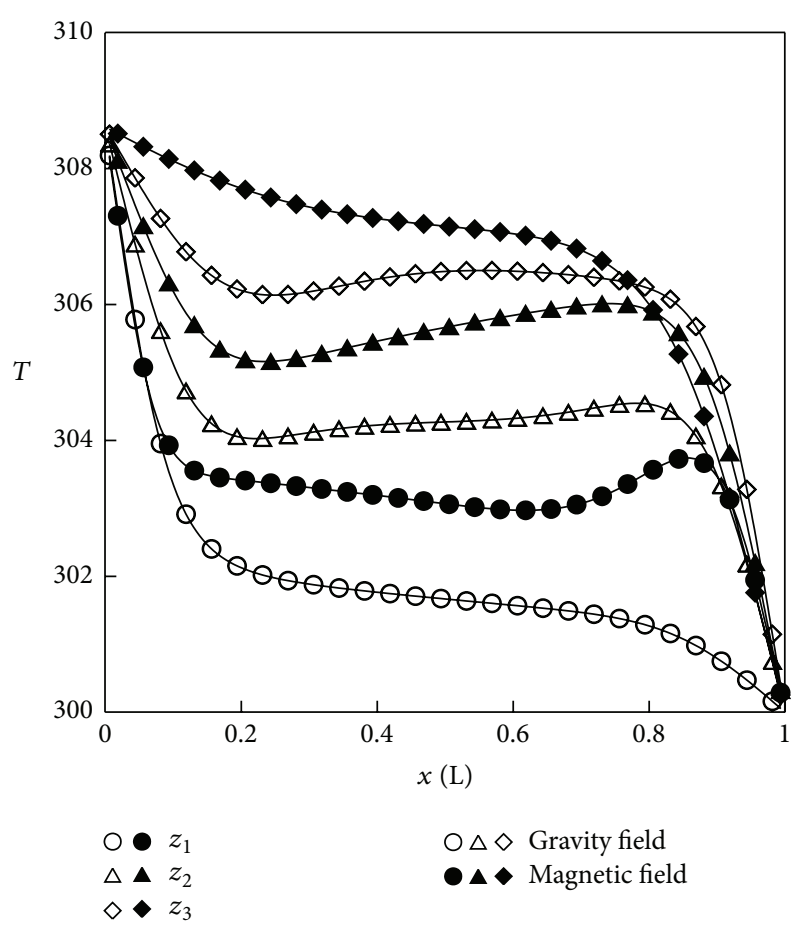

Figure 7: Temperature distribution along three lines parallel to $x$ axis.

TABLE 3: Summary of computed results.

\begin{tabular}{lcc}
\hline Model & $B_{r}$ & $\mathrm{Nu}$ \\
\hline & $0.5 \mathrm{~T}$ & 1.289 \\
& $1.5 \mathrm{~T}$ & 2.632 \\
Magnetic field & $2.5 \mathrm{~T}$ & 3.617 \\
& $3.5 \mathrm{~T}$ & 4.434 \\
& $4.5 \mathrm{~T}$ & 5.149 \\
\hline Gravity field & $g$ & 4.523 \\
\hline
\end{tabular}

that under the gravity field. As the magnetizing buoyancy force of air in the magnetic field is quite different from the gravitational buoyancy force, the natural convection in the enclosure under a magnetic field provided by a permanent magnet has quite different characteristics. For the model studied in this paper, the velocity and temperature fields are rotationally symmetric about the center of the enclosure. The heat transfer Nusselt numbers on the hot wall and the cold wall are symmetrical about the center line of the enclosure. But the velocity and temperature fields under the magnetic field do not have such characteristics. Heat transfer Nusselt number is different on the hot and cold walls. The value of local Nusselt number has a peak value on the bottom of the hot wall and decreases quickly along the hot wall, but the local Nusselt number changes with a relatively gentle rate on the cold wall. The peak value on the hot wall is three times larger than that under the gravity field when $B_{r}=4.5 \mathrm{~T}$ and 2 times larger when $B_{r}=2.5 \mathrm{~T}$. When $B_{r}$ is about $3.5 \mathrm{~T}$, the value of averaged $\mathrm{Nu}$ is nearly the same with that under gravity field. This means that in the zero gravity environment, a permanent magnet with $B_{r}=3.5 \mathrm{~T}$ can play a comparative role on the averaged Nusselt number compared with that under the gravity environment.

\section{Nomenclature}

A: Vector potential

B: Magnetic flux density (T)

$B_{r}$ : Residual magnetic flux density of a permanent magnet $(\mathrm{T})$

$\mathbf{f}_{m}$ : Magnetizing force $\left(\mathrm{N} / \mathrm{m}^{3}\right)$

$g$ : Acceleration coefficient of gravity $\left(\mathrm{m} / \mathrm{s}^{2}\right)$

H: Magnetic field strength $(\mathrm{A} / \mathrm{m})$

M: Magnetization

$\mathrm{Nu}$ : Nusselt number

$p$ : Pressure $\left(\mathrm{N} / \mathrm{m}^{2}\right)$

$p_{0}$ : Reference pressure without convection of gas $\left(\mathrm{N} / \mathrm{m}^{2}\right)$

$p^{\prime}$ : Perturbed pressure due to convection

Pr: Prandtl number

r: Unit vector

Ra: Rayleigh number

$t$ : Time (s)

T: $\quad$ Temperature $(\mathrm{K})$

u: Velocity vector $(u, w)(\mathrm{m} / \mathrm{s})$

$x$ : Coordinate $(\mathrm{m})$

$z$ : Coordinate $(\mathrm{m})$.

\section{Greek Symbols}

$\alpha$ : Thermal diffusivity of gas $\left(\mathrm{m}^{2} / \mathrm{s}\right)$

$\beta$ : Volume coefficient of expansion of gas due to temperature difference $(1 / \mathrm{K})$

$\mu$ : Viscosity of gas (Pa.s)

$\mu_{m}$ : Magnetic permeability of free space $(\mathrm{H} / \mathrm{m})$

$\nu: \quad$ Kinematic viscosity of gas $\left(\mathrm{m}^{2} / \mathrm{s}\right)$

$\rho:$ Density of gas $\left(\mathrm{kg} / \mathrm{m}^{3}\right)$

$\rho_{0}$ : Density of gas at a reference state of no convection $\left(\mathrm{kg} / \mathrm{m}^{3}\right)$

$\chi$ : Mass magnetic susceptibility of air $\left(\mathrm{m}^{3} / \mathrm{kg}\right)$

$\lambda$ : Conduction coefficient $(\mathrm{W} /(\mathrm{m} \cdot \mathrm{K}))$.

\section{Subscripts}

0: Reference state

$C$ : That of a cold wall

$h$ : That of a hot wall.

\section{Conflict of Interests}

The authors declare that there is no conflict of interests regarding the publication of this paper. 


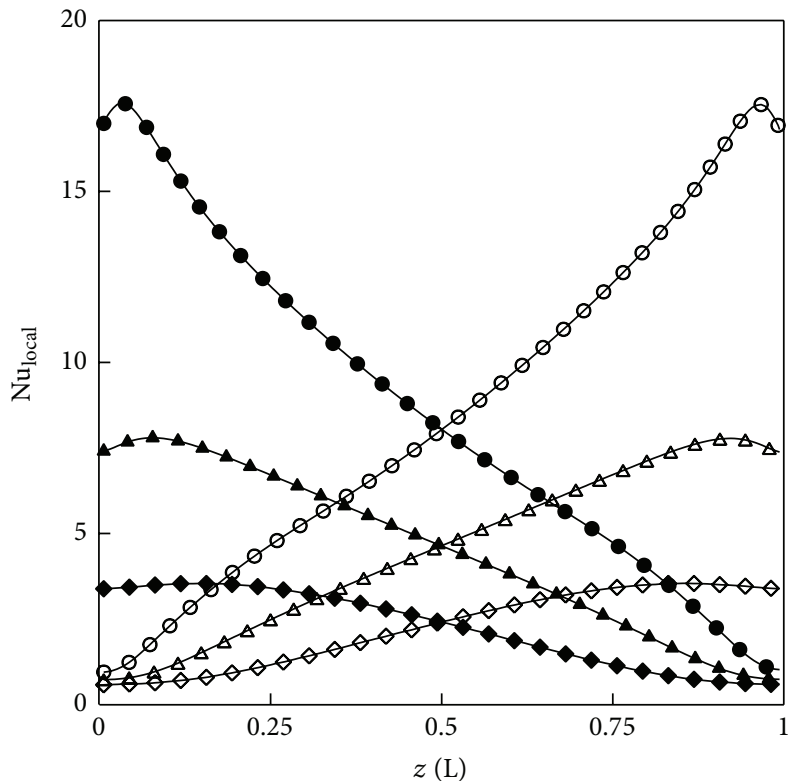

Gravity field
○ $\mathrm{Ra}=10^{6}$
$\triangle \Delta \mathrm{Ra}=10^{5}$
$\diamond \diamond \mathrm{Ra}=10^{4}$
$\mathrm{O} \diamond \diamond \mathrm{Nu}_{\text {local-c }}$
$\bullet \Delta \mathrm{Nu}_{\text {local-h }}$

(a)

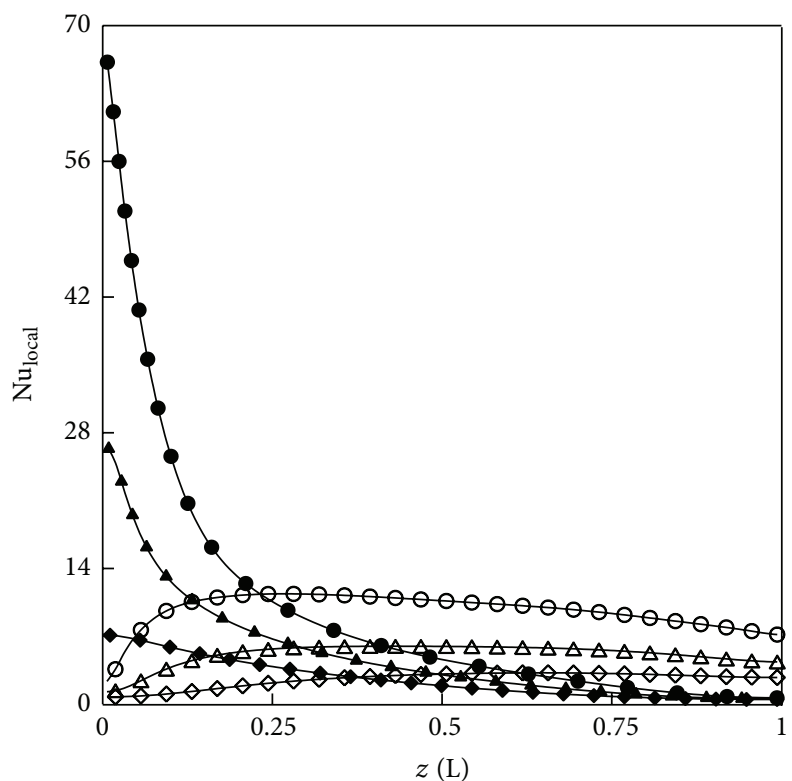

Magnetic field

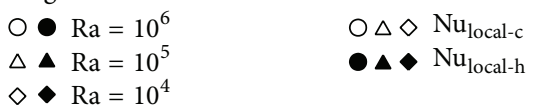

(b)

FIgURE 8: Distribution curves of $\mathrm{Nu}_{\text {local }}$ along the walls. (a) Under gravity field and (b) under magnetic field.

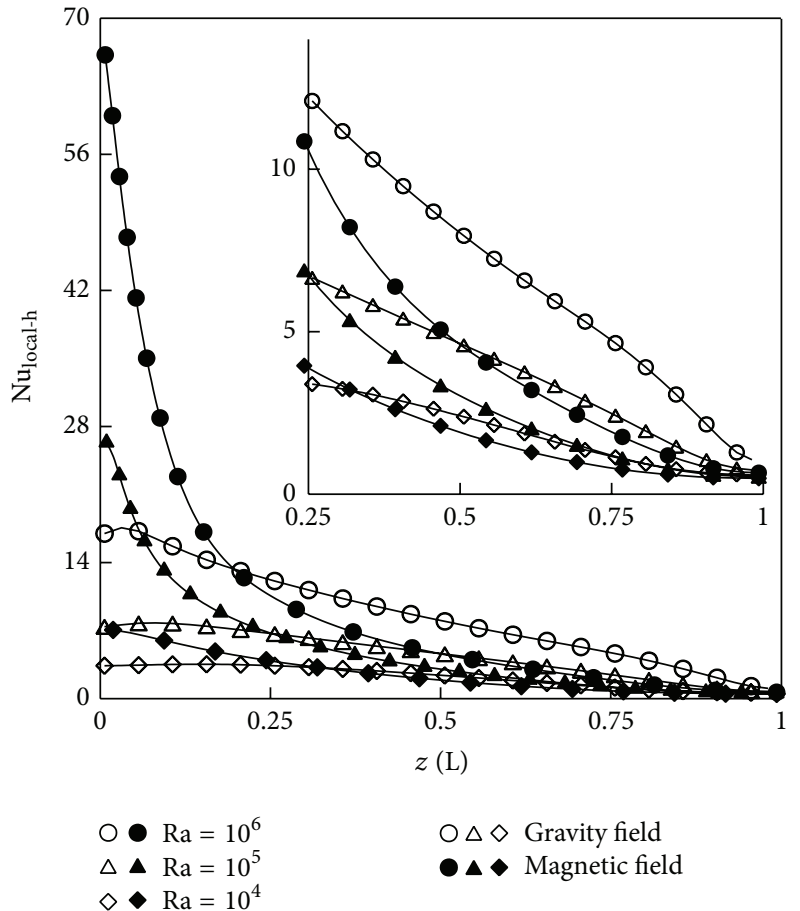

(a)

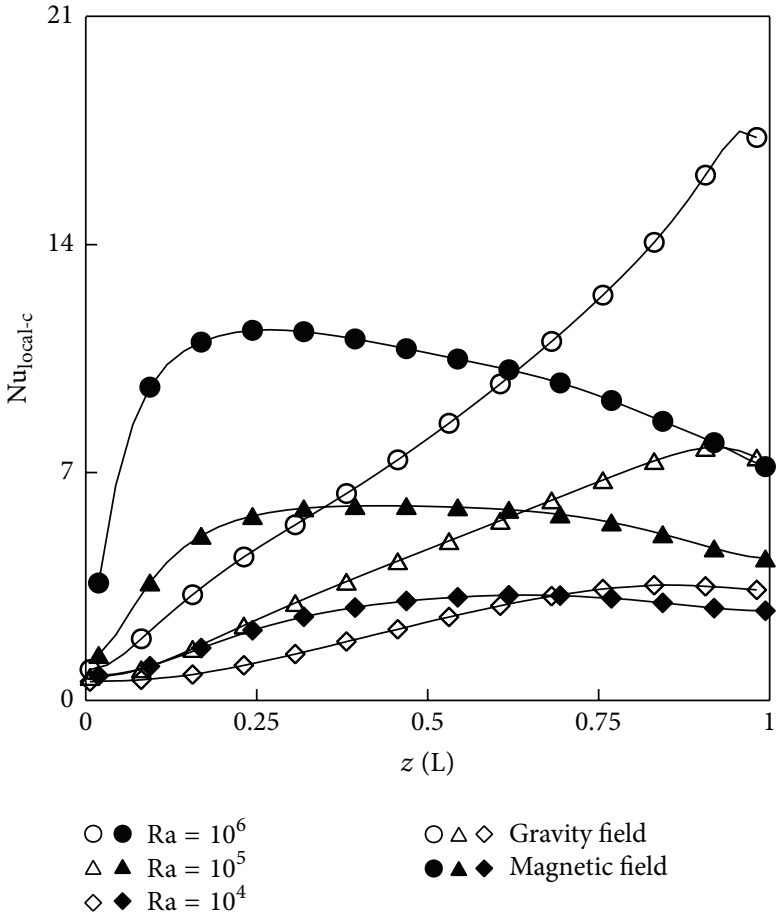

(b)

FIGURE 9: Comparison of $\mathrm{Nu}_{\text {local }}$ along the hot wall and cold wall. (a) Along hot wall and (b) along cold wall. 


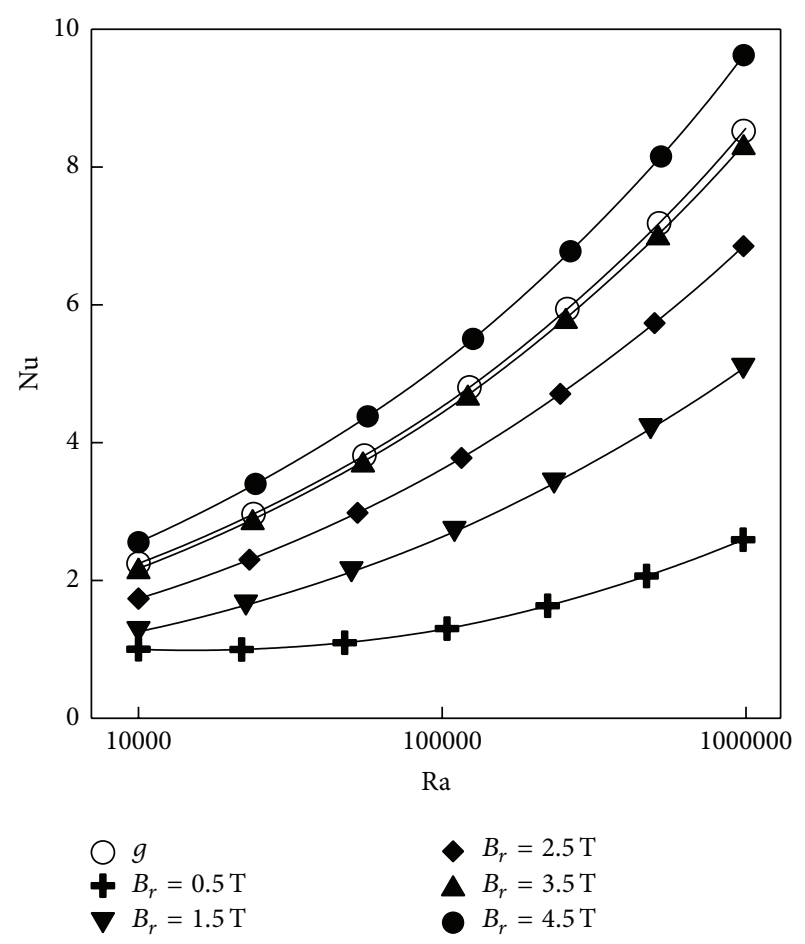

Figure 10: Comparison of the average Nusselt number.

\section{Acknowledgments}

This work is supported by Gansu Provincial Funds for Distinguished Young Scientists (no. 145RJDA324), the National Natural Science Foundation of China (no. 51376086), and the College Students' Innovative Experiment Project of Lanzhou Jiaotong University (20140048, 20140050).

\section{References}

[1] B. Gebhart, Y. Yaluria, R. L. Mahajan, and B. Sammakia, Buoyancy-Induced Flows and Transport, Hemisphere, Washington, DC, USA, 1988.

[2] K. T. Yang, "Natural convection in enclosures," in Handbook of Single Phase Convection Heat Transfer, Wiley, New York, NY, USA, 1987.

[3] G. de Vahl Davis, "Natural convection of air in a square cavity: a benchmark numerical solution," International Journal for Numerical Methods in Fluids, vol. 3, no. 3, pp. 249-264, 1983.

[4] C. J. Freitas, R. L. Street, A. N. Findikakis, and J. R. Koseff, "Numerical simulation of three dimensional flow in a cavity," International Journal for Numerical Methods in Fluids, vol. 5, no. 6, pp. 561-575, 1985.

[5] T. Fusegi, J. M. Hyun, K. Kuwahara, and B. Farouk, "A numerical study of three-dimensional natural convection in a differentially heated cubical enclosure," International Journal of Heat and Mass Transfer, vol. 34, no. 6, pp. 1543-1557, 1991.

[6] D. Braithwaite, E. Beaugnon, and R. Tournier, "Magnetically controlled convection in a paramagnetic fluid," Nature, vol. 354, no. 6349, pp. 134-136, 1991.
[7] N. I. Wakayama, "Behavior of gas flow under gradient magnetic fields," Journal of Applied Physics, vol. 69, no. 4, pp. 2734-2736, 1991.

[8] N. I. Wakayama, "Magnetic promotion of combustion in diffusion flames," Combustion and Flame, vol. 93, no. 3, pp. 207-214, 1993.

[9] N. I. Wakayama, "Magnetic acceleration and deceleration of $\mathrm{O}_{2}$ gas streams injected into air," IEEE Transactions on Magnetics, vol. 31, no. 1, pp. 897-901, 1995.

[10] N. I. Wakayama and M. Wakayama, "Magnetic acceleration of inhaled and exhaled flows in breathing," Japanese Journal of Applied Physics, vol. 39, no. 3, pp. L262-L264, 2000.

[11] O. Braithwaite, E. Beaugnon, and R. Tournier, "Magnetically controlled convection in a paramagnetic fluid," Nature, vol. 354, no. 6349, pp. 134-136, 1991.

[12] T. Tagawa, H. Ozoe, K. Inoue, M. Ito, K. Sassa, and S. Asai, "Transient characteristics of convection and diffusion of oxygen gas in an open vertical cylinder under magnetizing and gravitational forces," Chemical Engineering Science, vol. 56, no. 14, pp. 4217-4223, 2001.

[13] T. Tagawa, R. Shigemitsu, and H. Ozoe, "Magnetizing force modeled and numerically solved for natural convection of air in a cubic enclosure: effect of the direction of the magnetic field," International Journal of Heat and Mass Transfer, vol. 45, no. 2, pp. 267-277, 2002.

[14] M. Akamatsu, M. Higano, Y. Takahashi, and H. Ozoe, "Numerical computation on the control of aerial flow by the magnetizing force in gravitational and nongravitational fields," Numerical Heat Transfer A: Applications, vol. 43, no. 1, pp. 9-29, 2003.

[15] T. Bednarz, T. Tagawa, M. Kaneda, H. Ozoe, and J. S. Szmyd, "Convection of air in a cubic enclosure with an electric coil inclined in general orientations," Fluid Dynamics Research, vol. 36, no. 2, pp. 91-106, 2005.

[16] P. Filar, E. Fornalik, M. Kaneda, T. Tagawa, H. Ozoe, and J. S. Szmyd, "Three-dimensional numerical computation for magnetic convection of air inside a cylinder heated and cooled isothermally from a side wall," International Journal of Heat and Mass Transfer, vol. 48, no. 9, pp. 1858-1867, 2005.

[17] T. Bednarz, E. Fornalik, T. Tagawa, H. Ozoe, and J. S. Szmyd, "Experimental and numerical analyses of magnetic convection of paramagnetic fluid in a cube heated and cooled from opposing verticals walls," International Journal of Thermal Sciences, vol. 44, no. 10, pp. 933-943, 2005.

[18] T. P. Bednarz, C. Lei, J. C. Patterson, and H. Ozoe, "Effects of a transverse, horizontal magnetic field on natural convection of a paramagnetic fluid in a cube," International Journal of Thermal Sciences, vol. 48, no. 1, pp. 26-33, 2009.

[19] T. Bednarz, E. Fornalik, H. Ozoe, J. S. Szmyd, J. C. Patterson, and C. Lei, "Influence of a horizontal magnetic field on the natural convection of paramagnetic fluid in a cube heated and cooled from two vertical side walls," International Journal of Thermal Sciences, vol. 47, no. 6, pp. 668-679, 2008.

[20] T. Bednarz, T. Tagawa, M. Kaneda, H. Ozoe, and J. S. Szmyd, "Magnetic and gravitational convection of air with a coil inclined around the x axis," Numerical Heat Transfer A: Applications, vol. 46, no. 1, pp. 99-113, 2004.

[21] T. P. Bednarz, W. Lin, J. C. Patterson, C. Lei, and S. W. Armfield, "Scaling for unsteady thermo-magnetic convection boundary layer of paramagnetic fluids of $\mathrm{Pr}>1$ in micro-gravity conditions," International Journal of Heat and Fluid Flow, vol. 30, no. 6, pp. 1157-1170, 2009. 
[22] B. Bai, A. Yabe, J. Qi, and N. I. Wakayama, "Quantitative analysis of air convection caused by magnetic-fluid coupling," AIAA Journal, vol. 37, no. 12, pp. 1538-1543, 1999.

[23] R. B. Bird, W. E. Stewart, and E. N. Lightfoot, Transport Phenomena, John Wiley \& Sons, New York, NY, USA, 2007.

[24] P. Lorrain and D. R. Corson, Electromagnetic Fields and Waves, W.H. Freeman and Company, San Francisco, Calif, USA, 1970.

[25] S. V. Patankar, Numerical Heat Transfer and Fluid Flow, Hemisphere, New York, NY, USA, 1980. 


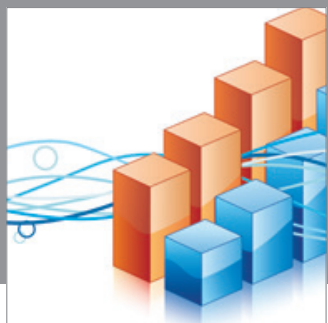

Advances in

Operations Research

mansans

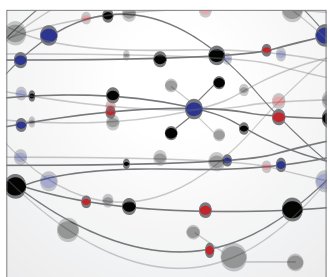

The Scientific World Journal
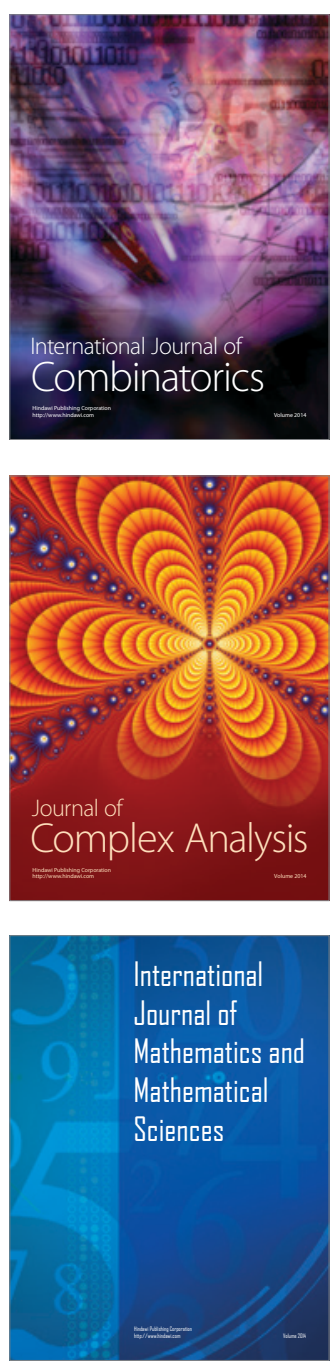
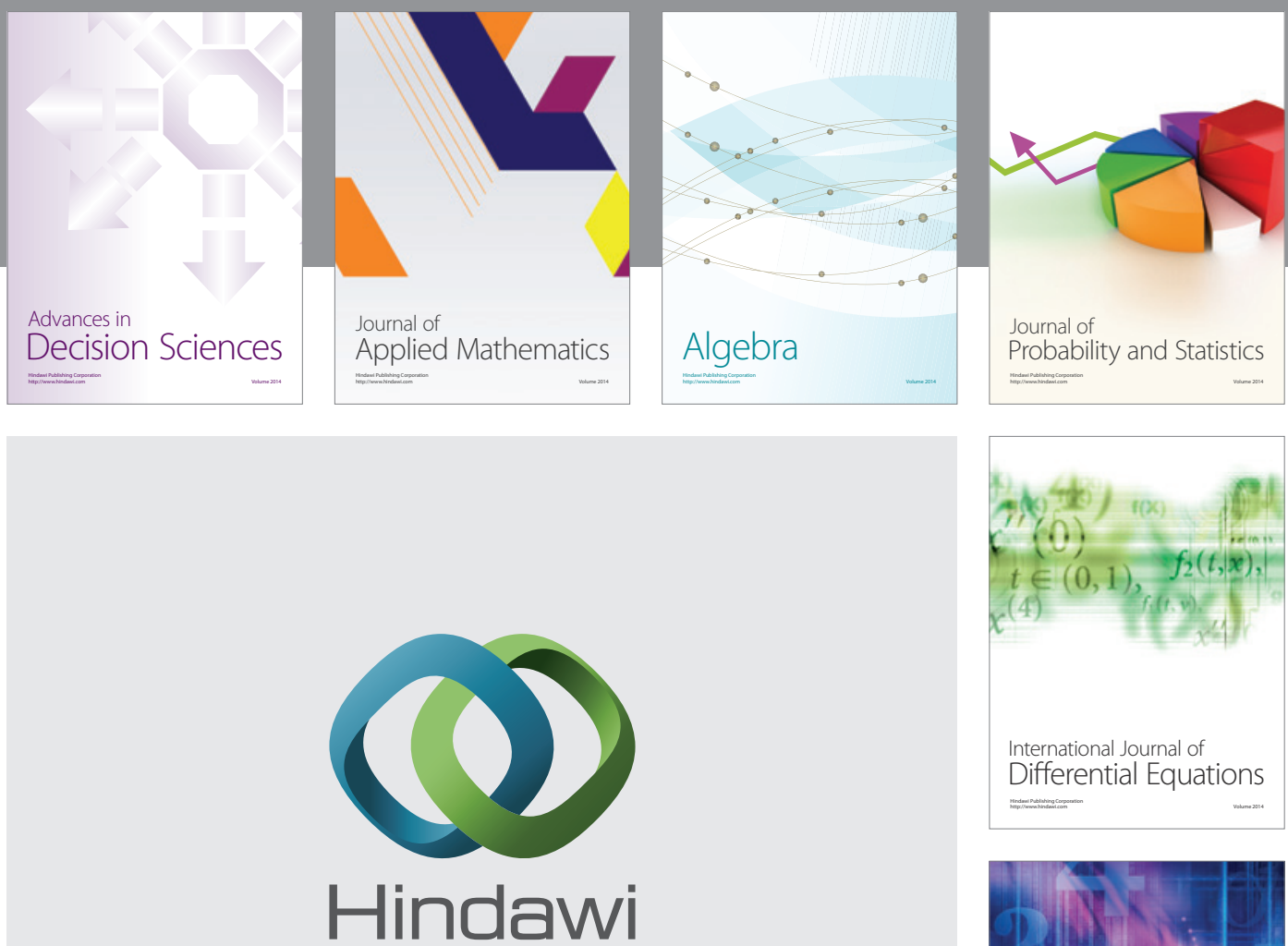

Submit your manuscripts at http://www.hindawi.com
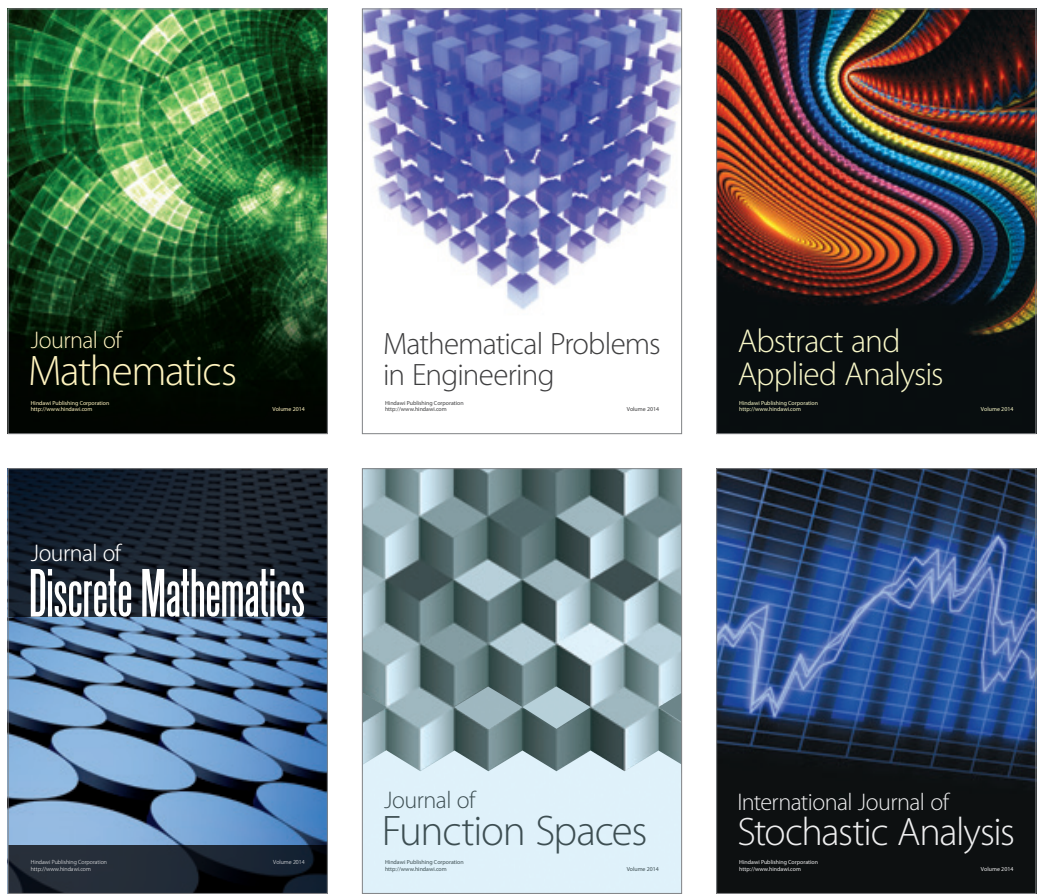

Journal of

Function Spaces

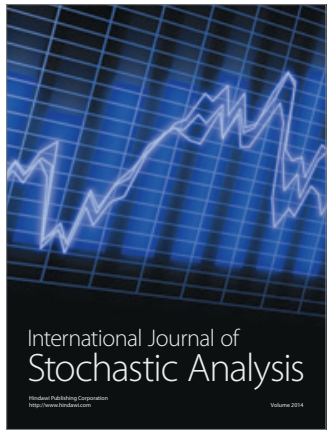

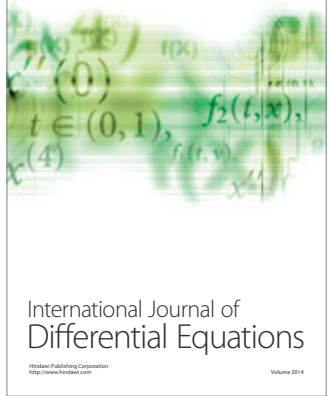
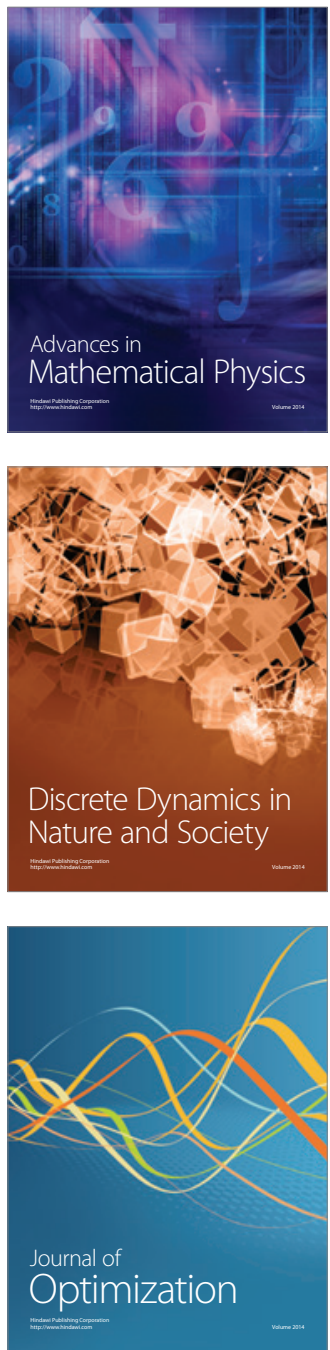